\title{
Treatment adherence and illness self-management: introduction to the special issue
}

\author{
M. Bryant Howren ${ }^{1,2} \cdot$ Jeffrey S. Gonzalez ${ }^{3,4}$
}

Received: October 3, 2016/Accepted: October 5, 2016/Published online: October 20, 2016

(C) Springer Science+Business Media New York (outside the USA) 2016

\begin{abstract}
The current issue is devoted broadly to research on treatment adherence and chronic illness self-management behavior. As the prevalence of chronic illness increases, the pervasive problem of treatment nonadherence is increasingly viewed as having a major impact on treatment outcomes, public health and healthcare costs, making this issue particularly timely. Sixteen articles spanning an array of topics are presented; articles include empirical studies, statistical simulations, systematic reviews, and theoretical commentaries. Studies conducted with diverse patient populations (e.g., chronic headache, diabetes, end-stage renal disease, HIV, hypertension, severe obesity), samples (e.g., adolescents, ethnic/racial minorities, low-income adults, parents, spousal dyads), and designs (e.g., cross-sectional, longitudinal assessment, randomized controlled trial), are represented. This issue highlights psychosocial factors associated with nonadherence, promising interventions to promote adherence, and state-of-the art methods for the study of illness self-management. We hope these articles engender even more high
\end{abstract}

The views expressed in this article are those of the authors and do not necessarily represent the views of the Department of Veterans Affairs.

M. Bryant Howren

matthew-howren@uiowa.edu

VA Iowa City Healthcare System, Iowa City, IA, USA

2 Department of Psychology, The University of Iowa, 11 Seashore Hall East, Iowa City, IA 52242, USA

3 Ferkauf Graduate School of Psychology, Yeshiva University, New York, NY, USA

4 Departments of Medicine and Epidemiology and Population Health, Albert Einstein College of Medicine, Bronx, NY, USA quality, methodologically rigorous research in this important subfield of behavioral medicine.

Keywords Treatment adherence - Illness self-management . Behavioral medicine $\cdot$ Health psychology $\cdot$ Special issue

The current issue of the Journal of Behavioral Medicine is devoted broadly to research and practice in treatment adherence and illness self-management. Nonadherence to treatment prescriptions and self-management regimens is rightfully recognized as an important contributor to global public health burden (Bosworth, 2010); consequently, research into factors associated with treatment (non)adherence, as well as interventions to improve adherence, has grown almost exponentially over the past three-plus decades. Despite enormous attention, however, poor adherence remains pervasive and is associated with a host of outcomes, including cost, patient and provider frustration, illness complications and relapse, increased hospitalization, poor health-related quality of life, and mortality (Christensen, 2004). Moreover, this constellation of behaviors also inhibits our ability to establish empiricallybased treatment guidelines. The impetus for this issue is rooted in the fact that behavioral medicine is particularly well suited to improve upon these outcomes and, as such, makes this issue particularly timely.

In response to an open call for papers on this topic, over 80 abstracts were submitted for consideration, of which approximately 45 were approved for full submission and peer-review. Multiple manuscript types (e.g., empirical articles, statistical simulations, systematic reviews, theoretical commentaries) focusing on diverse patient populations (e.g., chronic headache, diabetes, end-stage renal disease, HIV, hypertension, severe obesity), with numerous 
different participant samples (e.g., adolescents, ethnic/racial minorities, low-income adults, parents, spousal dyads), measured cross-sectionally and longitudinally, made the final cut; thus, 16 original articles covering several key issues in treatment adherence and illness self-management are presented here.

A number of review articles are included in this special issue. Leventhal et al. (2016) reviewed the development of the common sense model of self-regulation (CSM) framework over several decades, its application to acute and chronic health threats, and provide comments and recommendations for future research to maximize CSM's contribution to research aimed at improving the self-management of illness. Conn et al. (2016) conducted a systematic review and meta-analysis on blood pressure outcomes of medication adherence interventions across 156 studies and over sixty thousand participants. Another meta-analysis was conducted by Finitsis et al. (2016) analyzing the utility of the visual analogue scale (VAS) to capture antiretroviral adherence in patients with HIV/ AIDS. The authors examined data from 20 studies and described VAS concordance with other measures of medication adherence and viral load. Hood et al. (2016a) submitted a narrative review of evidence on adherence outcomes related to bariatric surgery in morbidly obese patients, highlighting the dearth of research associated with some outcomes in this area, as well as describing challenges and recommendations for improving self-management before and after surgery. Finally, a "review of reviews" was included which sought to synthesize existing evidence for mobile applications in the context of diabetes self-management (Hood et al., 2016b).

While several review articles are included, there are also a number of empirical studies, including trials of adherence interventions-both experimental and quasi-experimental in nature-and several which examined predictors or correlates of adherence behavior, particularly in the context of diabetes. Levine and colleagues (Levine et al., 2016) conducted a randomized trial of technology-assisted selfmonitoring of blood glucose in a sample of low-income older adults with type 2 diabetes. Another study (Berg et al., 2016) examined the relationship among adolescents', mothers', and fathers' reports of adolescents' adherence to the type 1 diabetes self-management regimen across six time-points, comparing these adherence ratings to measures of daily blood glucose and overall glycemic control through a multitrait-multimethod approach. In a sample of adults with type 2 diabetes, Phillips and colleagues (Phillips et al., 2016) tested behavioral initiation and maintenance factors derived from the CSM as correlates of adherence to medication and physical activity regimens. Additional studies in type 2 diabetes populations are also published in this issue: one examined dyadic data regarding various types of patient and spouse stressors as they relate to dietary and exercise adherence through mediating pathways involving depression symptoms and diabetesspecific self-efficacy (Anderson et al., 2016); another evaluated the efficacy of a text messaging/interactive voice response intervention on adherence and glycemic control in a sample of low-income adults with type 2 diabetes (Nelson et al., 2016); and one tested a number of constructs of modified social learning theory in relation to diabetes selfmanagement behaviors among type 2 diabetes patients on insulin regimens (Nugent \& Wallston, 2016).

Other studies of important patient populations are also included here. For example, Umeukeje and colleagues (Umeukeje, Merighi, Browne, et al., 2016) assessed endstage renal disease patients' perception of their healthcare providers' autonomy support in relation to phosphate binder medication adherence. Another examined race-based medical mistrust and medication beliefs in relation to antiretroviral medication adherence in a sample of HIV patients (Kalichman et al., 2016). Seng et al. (2016) developed and validated a theory-driven measure of selfefficacy for acute headache medication adherence, with input from both chronic headache patients and clinical headache experts in the scale development process.

A pair of articles dealt with issues related to measuring adherence. Tueller et al. (2016) used a simulation study to compare adherence outcomes when treated continuously and categorically, demonstrating considerable bias in parameter estimates and standard errors when treated dichotomously. Dunbar-Jacob and Rohay (2016) examined variation in predictors of adherence as a function of the method of measurement using data from two randomized controlled trials of adherence interventions.

These papers represent but a small cross-section of the promising work being conducted in the area of treatment adherence and illness self-management, but much more is yet to be done. Within this issue, Leventhal et al. (2016) highlight the need for a more nuanced understanding of our theoretical models, noting that concepts which have been traditionally understudied or ignored also must be given proper attention if we are to best explain the dynamic nature of adherence behavior and its underlying processes. Moreover, the rapid expansion of mHealth interventions underscores the need to test, refine, and apply theoretical models in all stages of intervention development and execution (Howren et al., 2013). It cannot be simply assumed that theoretical models applied in more traditional intervention settings "transfer" to such contexts. Two papers in the present issue are examples of theory-based mHealth interventions (i.e., Levine et al., 2016; Nelson et al., 2016).

We must also better elucidate provider-, family-, and system-level factors which may shape adherence behavior. The vast majority of existing research focuses on patient- 
level factors, for good reason. However, adherence may be compromised by many interpersonal, health system, social/ structural and cultural factors, in addition to those at the patient level. In this issue, Umeukeje et al. (2016) consider the importance of health care providers' support of patient autonomy, for example. Although providers were not directly queried, the implication is that provider practice characteristics may play an important role in adherence outcomes. In addition, Berg et al. (2016) assessed parent reports of adolescent adherence in type 1 diabetes, finding variation among fathers' and mothers' reports, which may represent a source of conflict and, ultimately, poor adherence. Their results suggest the need for interventions aimed at improving family communication which may increase adherence in adolescents. Finally, Anderson et al. (2016) demonstrated that stressors experienced by spouses, in addition to those experienced by patients with type 2 diabetes, can account for variation in important diabetes selfmanagement behaviors, at least partially through associated changes in depression symptoms and perceptions of competence for managing diabetes.

Accurate measurement of adherence and self-management behaviors remains a vexing issue with no straightforward resolution, some aspects of which are addressed by a pair of articles in the current issue (see also above; Dunbar-Jacob \& Rohay, 2016; Tueller et al., 2016). Fundamentally, the construct of adherence is not one behavior, but a complex of behaviors, and therefore is multiply determined. Behavior in one domain (e.g., diet) may not correlate well with behaviors in another domain (e.g., medication taking). Furthermore, research on various approaches to assessment and measurement has failed to produce a widely agreed upon 'gold-standard' for assessment. Even more objective assessment methods (e.g., pill counts vs. electronically-monitored dosing) are associated with measurement errors and bias (e.g., Gonzalez \& Schneider, 2011). As such, specific issues of measurement and assessment may differ across disease, time course, and patient, among other factors.

Use of multiple methods of measurement (e.g., self-report, electronically monitored) may improve the accuracy of adherence data (e.g., Llabre et al., 2006; Liu et al., 2001). Examination of relationships across methods of assessment also may provide useful information about the robustness and consistency of observed effects (e.g., Dunbar-Jacob \& Rohay, 2016). Research should seek to understand how best to capture the dynamic, heterogenous nature of adherence across time, whether by developing and validating new methods, refining existing ones or, perhaps more importantly, addressing broader conceptual issues which may present problems to empirical study. If adherence is truly a dynamic process that changes over time, single 'snapshot' assessments of adherence at a single point in time, whether assessed by subjective or objective measures, are unlikely to fully capture illness self-management behaviors and will likely account for minimal variance in disease outcomes. More rigorous assessment methods, such as repeated measurement and ecological momentary assessment (Shiffman et al., 2008), may better capture the dynamics of adherence and the factors that influence it over time. These methods should be used more routinely and may strengthen our evidence base.

In sum, many diverse patient populations and topic areas are represented in this issue, but several other important areas are not, in part due to the nature of an open request for submissions. Notably, however, of the eight core areas highlighted in the original call, seven are included here. ${ }^{1}$ We hope these articles are timely, useful, and engender even more high quality, methodologically rigorous research in this important subfield of behavioral medicine.

\section{References}

Anderson, J. R., Novak, J. R., Johnson, M. D., Dietz, S. L., Walker, A., Wilcox, A., et al. (2016). A dyadic multiple mediation model of patient and spouse stressors predicting patient dietary and exercise adherence via depression symptoms and diabetes selfefficacy. Journal of Behavioral Medicine. doi:10.1007/s10865016-9796-9.

Berg, C. A., Butner, J. E., Turner, S. L., Lansing, A. H., King, P., \& Wiebe, D. J. (2016). Adolescents', mothers', and fathers' reports of adherence across adolescence and their relation to HbA1c and daily blood glucose. Journal of Behavioral Medicine. doi:10. 1007/s10865-016-9771-5.

Bosworth, H. B. (2010). Improving patient treatment adherence: A clinician's guide. New York: Springer.

Christensen, A. J. (2004). Patient adherence to medical treatment regimens: Bridging the gap between behavioral science and biomedicine. Yale: Yale University Press.

Conn, V. S., Ruppar, T. M., \& Chase, J. D. (2016). Blood pressure outcomes of medication adherence interventions: Systematic review and meta-analysis. Journal of Behavioral Medicine. doi:10.1007/s10865-016-9730-1.

Dunbar-Jacob, J., \& Rohay, J. M. (2016). Predictors of medication adherence: Fact or artifact. Journal of Behavioral Medicine. doi:10.1007/s10865-016-9752-8.

\footnotetext{
${ }^{1}$ These core areas are as follows: (1) treatment adherence and chronic illness self-management (including health behavior change, selfmonitoring, and care-seeking); (2) care coordination efforts to improve treatment adherence; (3) methodological issues in measuring treatment adherence and self-management; (4) moderators of treatment adherence (e.g., patient, provider, and/or sociocultural factors); (5) new and emerging approaches to improving treatment adherence and self-management; (6) sociocultural considerations regarding treatment adherence and self-management; (7) treatment adherence and chronic illness self-management in special populations (e.g., ethnic minorities, children, the elderly, those with comorbid mental illness); (8) meta-analyses of relevant areas of the literature (predictors of adherence or outcomes of adherence).
} 
Finitsis, D. J., Pellowski, J. A., Huedo-Medina, T. B., Fox, M. C., \& Kalichman, S. C. (2016). Visual analogue scale (VAS) measurement of antiretroviral adherence in people living with HIV (PLWH): A meta-analysis. Journal of Behavioral Medicine. doi:10.1007/s10865-016-9770-6.

Gonzalez, J. S., \& Schneider, H. E. (2011). Methodological issues in the assessment of diabetes treatment adherence. Current Diabetes Reports, 11, 472-479.

Hood, M. M., Corsica, J., Bradley, L., Wilson, R., Chirinos, D. A., \& Vivo, A. (2016a). Managing severe obesity: Understanding and improving treatment adherence in bariatric surgery. Journal of Behavioral Medicine. doi:10.1007/s10865-016-9772-4.

Hood, M. M., Wilson, R., Corsica, J., Bradley, L., Chirinos, D. A., \& Vivo, A. (2016b). What do we know about mobile applications for diabetes self-management? A review of reviews. Journal of Behavioral Medicine. doi:10.1007/s10865-016-9765-3.

Howren, M. B., Van Liew, J. R., \& Christensen, A. J. (2013). Advances in patient adherence to medical treatment regimens: The emerging role of technology in adherence monitoring and management. Social \& Personality Psychology CompassHealth Series, 7, 427-443.

Kalichman, S. C., Eaton, L., Kalichman, M. O., Grebler, T., Merely, C., \& Welles, B. (2016). Race-based medical mistrust, medication beliefs and HIV treatment adherence: Test of a mediation model in people living with HIV/AIDS. Journal of Behavioral Medicine. doi:10.1007/s10865-016-9767-1.

Leventhal, H., Phillips, L. A., \& Burns, E. (2016). The CommonSense Model of Self-Regulation (CSM): A dynamic framework for understanding illness self-management. Journal of Behavioral Medicine. doi:10.1007/s10865-016-9782-2.

Levine, J. C., Burns, E., Whittle, J., Fleming, R., Knudson, P., Flax, S., et al. (2016). Randomized trial of technology-assisted selfmonitoring of blood glucose by low-income seniors: Improved glycemic control in type 2 diabetes mellitus. Journal of Behavioral Medicine. doi:10.1007/s10865-016-9763-5.

Liu, H., Golin, C. E., Miller, L. G., Hays, R. D., Beck, C. K., Sanandaji, S., et al. (2001). A comparison study of multiple measures of adherence to HIV protease inhibitors. Annals of Internal Medicine, 134, 968-977.

Llabre, M. M., Weaver, K. E., Durán, R. E., Antoni, M. H., McPherson-Baker, S., \& Schneiderman, N. (2006). A measurement model of medication adherence to highly active antiretroviral therapy and its relation to viral load in HIV-positive adults. AIDS Patient Care \& STDs, 20, 701-711.

Nelson, L. A., Mulvaney, S. A., Gebretsadik, T., Johnson, K. B., \& Osborn, C. Y. (2016). The MEssaging for Diabetes (MED) intervention improves short-term medication adherence among low-income adults with type 2 diabetes. Journal of Behavioral Medicine. doi:10.1007/s10865-016-9774-2.

Nugent, L. E., \& Wallston, K. A. (2016). Modified social learning theory re-examined: Correlates of self-management behaviors of persons with type 2 diabetes. Journal of Behavioral Medicine. doi:10.1007/s10865-016-9753-7.

Phillips, L. A., Cohen, J., Burns, E., Abrams, J., \& Renninger, S. (2016). Self-management of chronic illness: The role of 'habit' versus reflective factors in exercise and medication adherence. Journal of Behavioral Medicine. doi:10.1007/s10865-016-9732$\mathrm{z}$.

Seng, E. K., Nicholson, R. A., \& Holroyd, K. A. (2015). Development of a measure of self-efficacy for acute headache medication adherence. Journal of Behavioral Medicine. doi:10.1007/ s10865-015-9683-9.

Shiffman, S., Stone, A. A., \& Hufford, M. R. (2008). Ecological momentary assessment. Annual Review of Clinical Psychology, $4,1-32$.

Tueller, S. J., Deboeck, P. R., \& Van Dorn, R. A. (2016). Getting less of what you want: Reductions in statistical power and increased bias when categorizing medication adherence data. Journal of Behavioral Medicine. doi:10.1007/s10865-016-9727-9.

Umeukeje, E. M., Merighi, J. R., Browne, T., Wild, M., Alsmaan, H., Umanath, K., et al. (2016). Health care providers' support of patients' autonomy, phosphate medication adherence, race and gender in end stage renal disease. Journal of Behavioral Medicine. doi:10.1007/s10865-016-9745-7. 